\title{
Macrophage migration inhibitory factor protein and mRNA expression in cutaneous melanocytic tumours
}

\author{
CLELIA MIRACCO $^{1}$, MARIA CATERINA DE NISI ${ }^{1}$, FELICE ARCURI $^{1}$, ELENA COSCI $^{1}$, \\ LORENZO PACENTI $^{1}$, MARZIA TOSCANO ${ }^{1}$, ANNA VITTORIA LALINGA ${ }^{1}$, \\ MAURIZIO BIAGIOLI ${ }^{2}$, PIETRO RUBEGNI ${ }^{2}$, ROSELLA VATTI ${ }^{1}$, EMILIA MAELLARO ${ }^{3}$, \\ BARBARA DEL BELLO ${ }^{3}$, DANIELA MASSI ${ }^{4}$, PIETRO LUZI $^{1}$ and PIERO TOSI ${ }^{1}$
}

\begin{abstract}
${ }^{1}$ Department of Human Pathology and Oncology, Section of Pathological Anatomy, ${ }^{2}$ Department of Clinical Medicine and Immunological Sciences, Section of Dermatology, ${ }^{3}$ Department of Pathophysiology and Experimental Medicine, University of Siena; ${ }^{4}$ Department of Human Pathology and Oncology, Section of Pathological Anatomy, University of Florence, Italy
\end{abstract}

Received September 6, 2005; Accepted October 28, 2005

\begin{abstract}
Macrophage migration inhibitory factor (MIF) is a widely expressed cytokine involved in various biological processes. Although MIF's functions in cancer have not been completely elucidated, its expression has usually been correlated with tumour progression and aggressiveness, and it is currently discussed as a new promising target for novel therapies. Recent studies seem to confirm its active role in melanoma pathobiology; however, its expression has not yet been extensively studied in melanocytic tumours. We evaluated MIF protein expression in 126 skin lesions, including benign and atypical nevi, melanoma and melanoma metastases. In 55 cases, we also assessed MIF mRNA expression by realtime RT-PCR. Benign nevi were subdivided into nevocytic and Spitz/blue types; and melanomas into the radial, and vertical growth phase. A strong cytoplasmic MIF positivity was found in most samples, although it was more heterogeneous in malignant tumours; MIF nuclear expression characterized Spitz/blue nevi, atypical nevi, melanomas and metastases. All samples expressed MIF mRNA but it was significantly lower in benign nevi vs atypical nevi, melanomas and metastases ( $\mathrm{p}=0.001 ; \mathrm{p}<0.0001 ; \mathrm{p}=0.002$, respectively). Our study shows a widespread distribution of MIF among melanocytic tumours. Whereas we observed a trend towards higher expression levels of mRNA in atypical and malignant tumours, MIF protein was highly expressed in all lesions, although limited to the cytoplasm in most benign nevi. These observations suggest differences in MIF protein storage, subcellular location
\end{abstract}

Correspondence to: Dr Clelia Miracco, Sezione di Anatomia Patologica, Dipartimento di Patologia Umana e Oncologia, Università di Siena, Via delle Scotte 6, 53100 Siena, Italy E-mail:miracco@unisi.it

Key words: cutaneous melanocytic tumors, immunohistochemistry, macrophage migration inhibitory factor, real-time RT-PCR and properties in most benign nevi vs atypical and malignant tumours that should be confirmed by further investigation and correlation with clinical outcome.

\section{Introduction}

Macrophage migration inhibitory factor (MIF) was originally identified as a cytokine secreted by activated T-lymphocytes that was capable of preventing the random migration of macrophages (1). Thereafter, it has become evident that a broad spectrum of cells and tissue constitutively express MIF, and that it plays a role in a variety of biological processes, including regulation of the hypothalamo-pituitary-adrenal axis; cell proliferation and differentiation during wound repair; tissue remodeling; regulation of immune and inflammatory activities, including induction of inflammatory cytokines, nitric oxide and superoxide anion, and modulation of apoptosis and macrophage and lymphocyte proliferation (1-6). Furthermore, MIF is involved in angiogenesis, tumour growth, and acquired drug resistance (1,3,7-10). MIF also induces immunotolerance by inhibiting NK cell activity $(4,11)$. MIF seems capable of activating different signalling pathways, depending on cell type and induction state, as it happens for many other cytokines (12-19). It is supposed to be an evolutionarily conserved protein with multiple functions and, as such, it fueled interest as a potential clinical target for cancer and non-cancer therapies (4). Although it is an object of investigation in various types of tumours, to date there is no complete agreement in the literature on its functions in cancer (20-30). Probably, depending on the cell types and context, MIF can play contradictory roles, for instance proliferation or inhibition (6). MIF protein and its mRNA have been investigated in some human cancer types, and an array of recent reports indicates MIF as a critical mediator of tumourigenesis, mainly on the basis of the correlation between MIF expression levels and tumour occurrence and progression, as well as on the potent antitumour activity of anti-MIF Ab treatment and MIF gene silencing (6-9,19,24, 26-28,31). Whereas some authors suggest that MIF may not act in a proliferative manner directly but indirectly through growth factors, a direct involvement of MIF in cell cycle 
control has been claimed by others (3,12-14,16-19). Furthermore, whereas some authors suggest that MIF is mainly involved in the initial phase of tumourigenesis, its role in later tumour stages is becoming evident (12-15). MIF has been found to promote angiogenesis and the invasiveness and matrix degrading activity of cancer cells by stimulating matrix metalloproteinase; furthermore, it participates in the complex interactions between tumour cells and mononuclear cells, including $\mathrm{T}$ lymphocytes and macrophages $(19,32,33)$. MIF preservation of macrophage viability within the tumour may promote its progression and metastatic potential. Furthermore, MIF seems capable of enhancing macrophage cytotoxicity and angiogenic chemokine secretion $(3,15,26)$. Many studies on MIF involvement in cancer have been performed in vitro. There is a need to increase investigation on MIF in tumours in vivo, and some studies on biopsies from various tumour types are providing further insight into the role of MIF in cancer (20-22,24-28,30). Increasing MIF expression has usually been linked with tumour progression and correlated to a poor impact on prognosis (3,6-9,20-28,30-32). A prevalently cytoplasmic expression of MIF has usually been found in the human cancer types studied until now, although the biological significance of MIF subcellular localization is still unclear $(7,17,20-28,30,36,37)$. To our knowledge, MIF expression has not been extensively studied in melanocytic tumours in vivo. In this study, we analysed the expression of MIF protein and its mRNA in a series of benign and malignant cutaneous melanocytic lesions, by immunohistochemistry and quantitative real-time RT-PCR.

\section{Materials and methods}

One hundred and twenty-six consecutive cutaneous melanocytic lesions, in which there was diagnostic consent among three independent pathologists (CM; MCDN; AVL), entered our study. There were 40 benign nevi, including 30 compound nevocytic nevi, 8 compound Spitz nevi and 2 blue nevi; 20 atypical nevi (AN), including 2 lesions removed from two patients with dysplastic nevus syndrome and 18 sporadic AN; 54 melanomas; and 12 cutaneous melanoma metastases. Thirtyone melanomas were in the radial growth phase (RGP), ranging in thickness from 0 to $0.30 \mathrm{~mm}$ (median: $0.22 \mathrm{~mm}$ ). Twentythree melanomas were in the vertical growth phase (VGP), ranging in thickness from 0.40 to $3.5 \mathrm{~mm}$ (median: $2.1 \mathrm{~mm}$ ). In 2 RGPMs and 8 VGPMs, there were remnants of a benign nevus at one periphery of the lesion. In 55 cases (10 RGP and 10 VGP melanomas; 2 atypical nevi of two patients with dysplastic nevus syndrome; 8 sporadic atypical nevi; 6 metastases; 8 Spitz nevi; and 11 compound nevocytic nevi), at the surgical remotion of the lesion, some fragments from the tumour and tumour-free skin margins were immediately harvested and frozen in liquid $\mathrm{N}_{2}$ for gene expression analysis.

Immunohistochemistry. Immunohistochemistry of MIF was performed using the streptavidin-biotin method as described (37). Paraformaldehyde-fixed and paraffin-embedded tissues were cut into $4-\mu \mathrm{m}$ sections, deparaffinized in xylene, and passed through decreasing concentrations of alcohol in water. After washing in Tris-buffered saline ( $\mathrm{pH} 7.6, \mathrm{TBS})$, they were incubated with normal rabbit serum (Dako, Copenhagen,
Denmark) diluted 1:5 in TBS to prevent non-specific binding, and excess serum was removed by blotting. Anti-human MIF goat polyclonal antibody (R\&D Systems, Abingdon, UK) was diluted 1:400 in TBS and applied to the sections, and incubation was carried out overnight at $4^{\circ} \mathrm{C}$. Slides were then washed three times with TBS for $5 \mathrm{~min}$ and incubated with rabbit anti-goat antibody labeled with peroxidase (Calbiochem, Milan, Italy) at a dilution of 1:2000 for 30 min. After being washed three times for $5 \mathrm{~min}$ in TBS, sections were stained with new fuchsin as chromogenic substrate and counterstained with Meyer's hematoxylin. The reaction was stopped by washing the sections in distilled water, and slides were mounted and observed under a light microscope. For each case, a negative control was obtained by replacing the specific antibody with non-immune serum immunoglobulins at the same concentration as the primary antibody. Positivity to MIF was revealed by a red staining.

Assessment of immunostaining. Both nuclear and cytoplasmic staining were evaluated by two independent pathologists (CM; MCDN), counting up to 1000 cells in randomly chosen fields. A simultaneous reassessment of scoring was performed in case there were different results. Nuclear immunostaining was considered positive if $>10 \%$ of tumour cell nuclei were stained by the antibody, independently on the degree of positivity, and a cut-off of $50 \%$ was established between cases showing low $(\leq 50 \%)$ or high $(>50 \%)$ nuclear MIF expression. Cytoplasmic positivity was scored by both the intensity $(0$ negative to 3 strong) and percentage of cells stained (1, $\leq 50 \%$; $2,>50 \%$ ); a score from 2 to 5 was assigned to positive cases.

\section{Gene expression analysis}

RNA isolation and cDNA preparation. Total RNA was extracted from frozen fragments and purified using Trizol reagent (Invitrogen, Milan, Italy) according to the manufacturer's protocol. The quality of the RNA samples was determined by electrophoresis through agarose gels and staining with ethidium bromide, and the $18 \mathrm{~S}$ and $28 \mathrm{~S}$ RNA bands were visualized under ultraviolet light. The concentration of RNA was calculated by measuring the absorbance at $260 \mathrm{~nm}$. In each sample, $5 \mu \mathrm{g}$ of RNA were treated with DNAse, by using the RQ1 RNase-Free DNase kit (Promega, Florence, Italy). For cDNA synthesis, RNA (500 ng) was reverse transcribed in a final volume of $20 \mu \mathrm{l}$ containing $4 \mu \mathrm{l}$ of $5 \mathrm{X}$ First-Strand Buffer, $1 \mu 1$ of dNTPs $10 \mathrm{mM}, 0.6 \mu 1$ (24 units) of Rnasis RNase inhibitor (Promega), $2 \mu 1$ of DTT $0.1 \mathrm{M}, 1 \mu \mathrm{l}$ (200 units) of M-MLV Reverse Transcriptase (Invitrogen), $0.5 \mu 1$ (200 ng) random hexamers (Pharmacia, Milan, Italy). The samples were incubated at $25^{\circ} \mathrm{C}$ for $10 \mathrm{~min}$, and $37^{\circ} \mathrm{C}$ for $50 \mathrm{~min}$. Reverse transcriptase was then inactivated by heating at $70^{\circ} \mathrm{C}$ for $15 \mathrm{~min}$. No-RT controls were perfomed by omitting the addition of the reverse transcriptase enzyme, and no-template controls were performed by the addition of nuclease-free water. All products were stored at $-20^{\circ} \mathrm{C}$ for future use.

Quantitative real-time $R T$-PCR. A relative quantitation of MIF mRNA expression normalized to three endogenous reference genes (ß-actin, BACT; glyceraldehyde-3-phosphate-dehydrogenase, GAPDH; hypoxanthine ribosyltransferase, HPRT), as suggested by the literature (38), was performed by real-time 
Table I. Primer, probe sequences and size of amplification products.

\begin{tabular}{lll}
\hline & \multicolumn{1}{c}{ Sequence } & Product (bp) \\
\hline HPRT sense & 5'-TGCTGAGGATTGGAAAGG-3' & 113 \\
HPRT anti-sense & 5'-AGAGGGCTACAATGTGATGG-3' \\
HPRT quantiprobe & 5'-TGAAGGAGATGGGAGG-3' \\
BACT sense & 5'-ATTGGCAATGAGCGGTTC-3' \\
BACT anti-sense & 5'-ATGTCCACGTCACACTTC-3' \\
BACT quantiprobe & 5'-TTCCAGCCTTCCTTCC-3' \\
GAPDH sense & 5'-GTCTCCTCTGACTTCAACA-3' \\
GAPDH anti-sense & 5'-CAGGAAATGAGCTTGACAAA-3' \\
GAPDH quantiprobe & 5'-ACCCACTCCTCCACCT-3' \\
MIF sense & 5'-GGACAGGGTCTACATCAA-3' \\
MIF anti-sense & 5'-TTAGGCGAAGGTGGAGTT-3' \\
MIF quantiprobe & 5'-TTACGACATGAACGCGG-3' \\
\hline
\end{tabular}
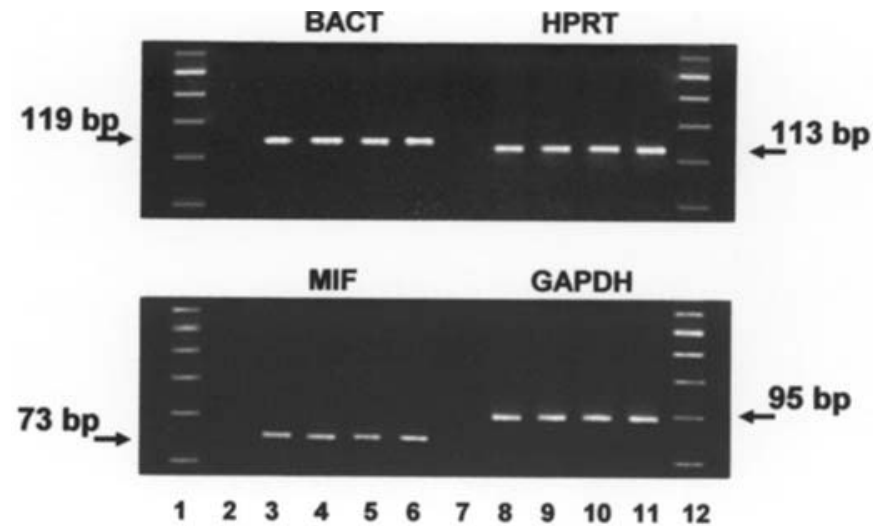

Figure 1. RT-PCR analysis of gene mRNA. The PCR products were electrophoresed on $2 \%$ agarose gel. MIF, 73 bp; glyceraldehyde-3-phosphatedehydrogenase (GAPDH), 95 bp; hypoxanthine ribosyltransferase (HPRT), $113 \mathrm{bp}$; B-actin (BACT), $119 \mathrm{bp}$. Lanes 1 and 12, the molecular size marker; lanes 3 and 8 , a benign nevocytic nevus; lanes 4 and 9, an atypical nevus; lanes 5 and 10, a vertical growth phase melanoma; lanes 6 and 11, a melanoma metastasis.

reverse transcription PCR (RT-PCR), using the DNA Engine Opticon 2 system (MJ Research, Celbio, Milan, Italy) and the QuantiProbes detection system (Qiagen GmbH, Milan, Italy). The real-time PCR method is based on the detection and quantitation of a fluorescent reporter (39). A fluorescence signal is generated which increases in direct proportion to the amount of PCR product. Primer, probe sequences, and the size of amplification products of all tested genes are listed in Table I; all primers and probes were prepared using QuantiProbe design software (Qiagen) and synthesized by Qiagen. The PCR reaction mixture (final volume, $20 \mu \mathrm{l}$ ) contained $10 \mu \mathrm{l}$ of 2X QuantiTect Probe Master mix (Qiagen), $1 \mu 1$ of 20X Primers mix (Qiagen), $1 \mu 1$ of 20X QuantiProbe solution (Qiagen), $1 \mu \mathrm{l}$ of c-DNA and $7 \mu \mathrm{l}$ of water. Thermocycling conditions were $15 \mathrm{~min}$ at $95^{\circ} \mathrm{C}$, followed by 45 cycles of denaturation $\left(94^{\circ} \mathrm{C}, 15 \mathrm{sec}\right)$, annealing $\left(56^{\circ} \mathrm{C}, 30 \mathrm{sec}\right)$ and extension $\left(76^{\circ} \mathrm{C}, 30 \mathrm{sec}\right)$. Gene products on a gel are depicted in Fig. 1. All samples were analyzed in triplicate wells of a 96-well plate. The results of real-time RT-PCR data were represented by the parameter $\mathrm{Ct}$ (threshold cycle number), where $\mathrm{Ct}$ is defined as the PCR fractional cycle number at which the fluorescence emission exceedes a threshold fixed in the range of linearity of a standard dilution curve produced contemporary to the run of samples. The higher the starting copy number of the nucleic acid target, the sooner a significant increase in fluorescence is observed; therefore, the lowest $\mathrm{Ct}$ values correspond to the highest nucleic acid starting copy number. Ct was calculated for MIF and normalized to the median $\mathrm{Ct}$ value of the reference genes. Briefly, the MIF gene expression value was expressed by $2^{\Delta \mathrm{Ct}}$, obtained by using a slightly modified $2^{-\Delta \Delta C t}$ data analysis method (39) where $\Delta \mathrm{Ct}$ is the difference obtained between the $\mathrm{Ct}$ value of MIF and the median $\mathrm{Ct}$ value of the reference genes. The highest $2^{\Delta \mathrm{Ct}}$ values correspond to the lowest MIF nucleic acid copy number in the sample.

Data analysis. Median values of $\Delta \mathrm{Ct}$ were compared by using the Mann-Whitney U-test. Statistical significance was set at $\mathrm{p}<0.05$.

\section{Results}

MIF protein. A widespread distribution of MIF protein was observed in all samples. The basal layers of epidermis and adnexa showed a mild to moderate cytoplasmic positivity to MIF; in addition, some basal cells also showed a nuclear positivity. A mild to moderate cytoplasmic positivity to MIF was also observed in nerves, smooth muscle and vessel endothelium. When present, mononucleated inflammatory cells including lymphocytes and macrophages at the periphery of the lesion showed a moderate to strong cytoplasmic or nucleo-cytoplasmic positivity to MIF. Usually, intratumoural lymphocytes were positive to MIF, whereas intratumoural macrophages were negative. The cytoplasm of fat cells was either negative or weakly positive to MIF. The distribution of MIF expression in melanocytes and their tumours by histology 


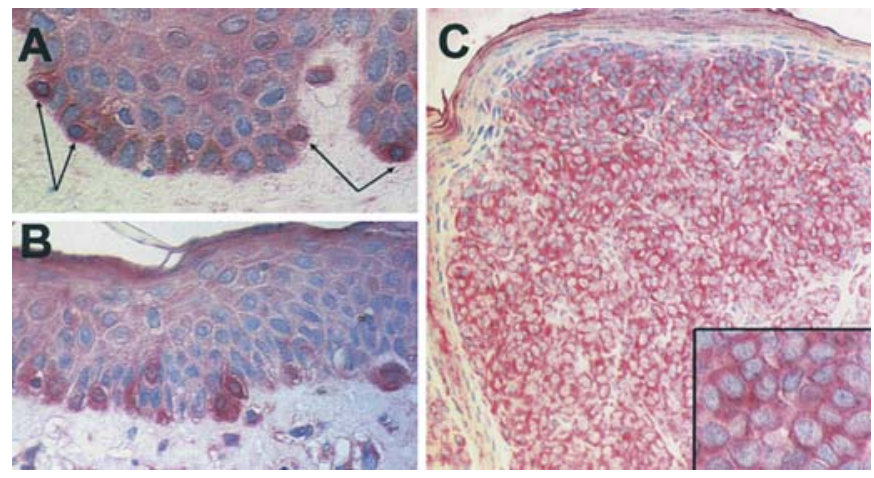

Figure 2. Predominantly cytoplasmic MIF expression in normal melanocytes (A, arrows), and in a compound benign nevocytic nevus (C). Nucleo-cytoplasmic positivity to MIF in the junctional component of a benign common nevus (B). Epidermal cells also express MIF protein in their cytoplasm. Streptavidin-biotin: substrate, new fuchsin; original magnification: A, B, C inset, $\mathrm{x} 400 ; \mathrm{C}, \mathrm{x} 200$.

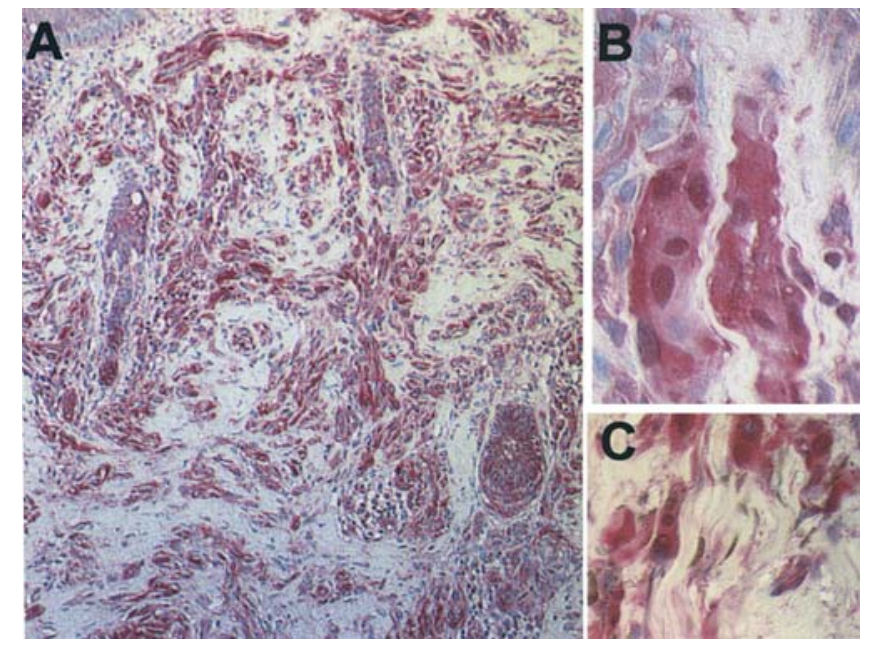

Figure 3. MIF staining of tumour cell cytoplasms and nuclei in a Spitz (A and $\mathrm{B})$ and in a blue nevus $(\mathrm{C})$. Streptavidin-biotin: substrate, new fuchsin; original magnification: A, x50; B and C, x400.

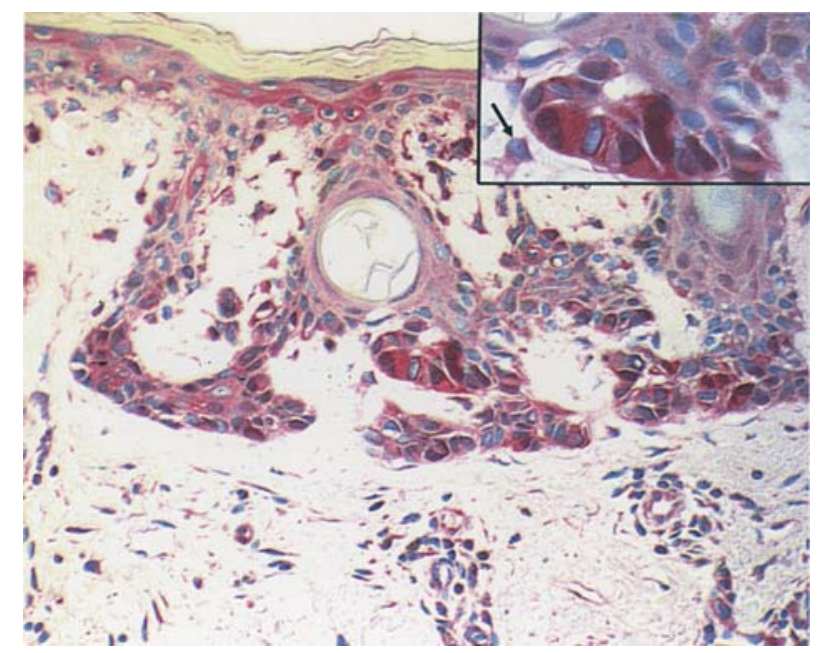

Figure 4. Nucleo-cytoplasmic positivity of melanocytes in an atypical nevus; the inset shows atypical junctional melanocytes and a macrophage (arrow) with MIF-positive cytoplasm in the dermis. Streptavidin-biotin: substrate, new fuchsin; original magnification: $\mathrm{x} 200$; inset, $\mathrm{x} 400$.
Table II. Melanocytic tumours and their immunoreactivity to MIF antibody.

\begin{tabular}{lcrrrrrr}
\hline & & \multicolumn{1}{c}{ Nuclear (+) } & \multicolumn{5}{c}{ Cytoplasmic (+) } \\
Cases & NC- & no. $(\%)$ & 2 & 3 & 4 & 5 \\
\hline CN (30) & 0 & 0 & & 0 & 0 & 0 & 30 \\
SN (8) & 0 & 3 & $(25)$ & 0 & 0 & 0 & 8 \\
& & 5 & $(50)$ & & & & \\
BIN (2) & 0 & 1 & $(25)$ & 0 & 0 & 0 & 2 \\
AN (20) & 0 & 6 & $(25)$ & 0 & 0 & 0 & 20 \\
& & 14 & $(>50)$ & & & & \\
RGPM (31) & 0 & 8 & $(30)$ & 0 & 0 & 0 & 31 \\
& & 20 & $(>50)$ & & & & \\
VGPM (23) & 1 & 2 & $(30)$ & 0 & 2 & 0 & 20 \\
& & 16 & $(>50)$ & & & & \\
MMet (12) & 1 & 6 & $(30)$ & 0 & 0 & 0 & 11 \\
& & 4 & $(>50)$ & & & & \\
\hline
\end{tabular}

Nucleo-cytoplasmic negative cases $\left(\mathrm{NC}^{-}\right)$, the number (no.) of cases showing nuclear positivity $>10 \%(+)$, the percentage of positive nuclei $(\%)$, and the number of cases subdivided on the basis of scored cytoplasmic positivity (2-5) are reported. CN, compound nevocytic nevi; SN, Spitz nevi; BIN, blue nevi; AN, atypical nevi; RGPM, radial growth phase melanoma; VGPM, vertical growth phase melanoma; MMet, melanoma metastases.

is shown in Figs. 2-5 and Table II. The cytoplasm of normal melanocytes was moderately to strongly positive to MIF, whereas the nucleus was either negative or weakly positive in $<10 \%$ of melanocytes. Among melanocytic tumours, all benign nevi showed a homogeneous, strong, cytoplasmic positivity to MIF (score 5) throughout the lesion. Whereas, in compound nevocytic nevi, nuclear staining was negative, all Spitz nevi showed nuclear MIF expression and it was high in 5 out of 8 cases $(62,2 \%)$. One out of the two analysed blue nevi showed a low nuclear MIF expression. A nucleo-cytoplasmic positivity characterized all atypical nevi and almost all melanomas and metastases. However, in melanomas, expecially in the VGP group, MIF positivity was often heterogeneous, showing either alternating negative and positive areas or negative cells intermingled with positive cells and/or with cells showing either cytoplasmic or nuclear positivity; positive nuclei were hazardly distributed throughout the lesion. In metastases, MIF positive nuclei were usually more numerous at the periphery of the lesion. All atypical nevi showed a strong cytoplasmic positivity (score 5), and a high nuclear expression was found in 14 out of 20 cases $(70 \%)$. Among malignant tumours, one out of $23(4.9 \%)$ VGP melanomas and one out of $12(8.3 \%)$ metastases were completely negative for MIF. As for cytoplasmic positivity, 51 out of $54(94 \%)$ melanomas, including 31 out of 31 (100\%) RGP melanomas and 20 out of $23(86,9 \%)$ VGP melanomas, scored 5. Score 5 was also assigned to 11 out of $12(90.1 \%)$ metastases. As for nuclei, 36 out of $54(66.6 \%)$ melanomas, including 20 out of $31(64.5 \%)$ RGP and 16 out of $23(69 \%)$ VGP melanomas, showed a high MIF expression, 


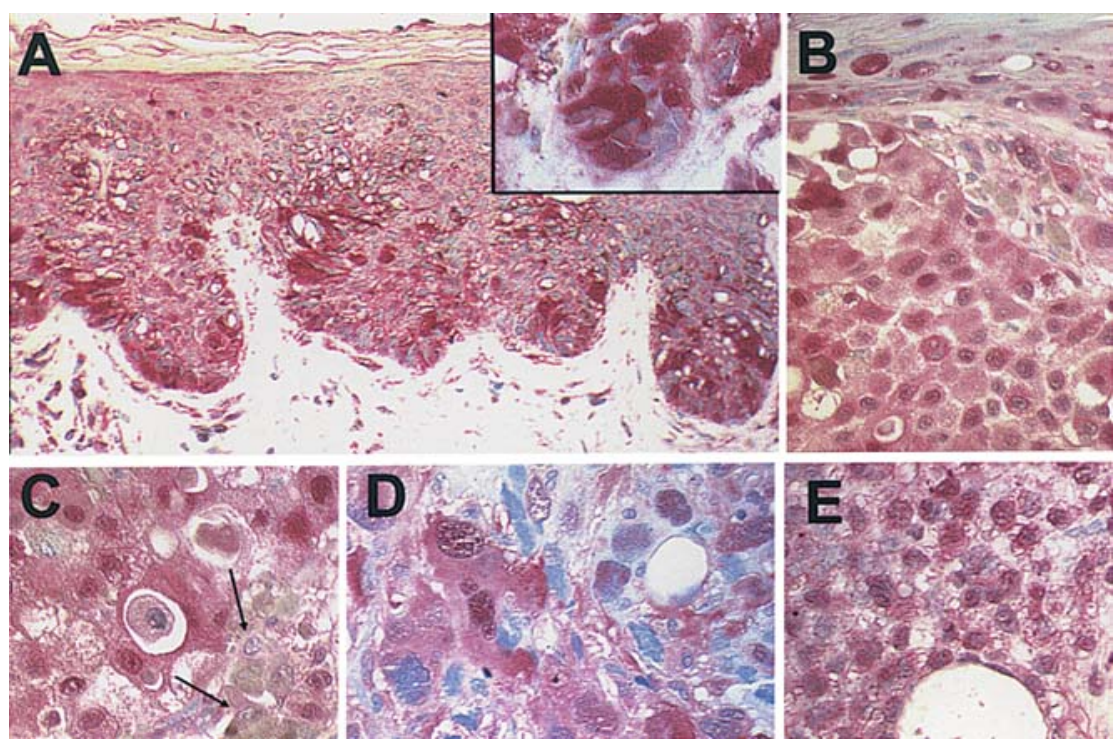

Figure 5. An acral lentiginous RGP melanoma showing nucleo-cytoplasmic positivity to MIF (A). Nucleo-cytoplasmic positivity to MIF in a VGP superficial spreading melanoma (B and C); notice intratumoural macrophages negative to MIF (arrows). Heterogeneous expression of MIF in another VGPM showing a mixture of negative cells, nucleo-cytoplasmic positive cells, and cells with either cytoplasmic or nuclear positivity to MIF (D). Predominantly nuclear positivity to MIF in a melanoma metastasis (E). Streptavidin-biotin: substrate, new fuchsin; original magnification: A, x100; A inset, B-E, x400.

Table III. Median values of $2^{\Delta \mathrm{Ct}}$ in each group of lesions and in the corresponding non-lesional skin. Range of values in brackets.

\begin{tabular}{lcccc}
\hline & $\mathrm{CN}+\mathrm{SN}$ & $\mathrm{CN}$ & $\mathrm{SN}$ & AN \\
\hline ML & 2755 & 2755 & 2722 & 457 \\
& $(270.5-9421)$ & $(270.5-9421)$ & $(1089-6154)$ & $(30.6-6672)$ \\
N-LS & 120400 & 69860 & 78210 & 10824 \\
& $(8364-234020)$ & $(8364-130600)$ & $(92125-234020)$ & $(9540-98222)$ \\
\hline & RGPM + VGPM & RGPM & VGPM & MMet \\
\hline ML & 579 & 712 & 553 & 588 \\
& $(123-40400)$ & $(123-40400)$ & $(279-1530)$ & $(221-1475)$ \\
N-LS & 12826 & 12826 & 9022 & 13012 \\
& $(8240-210523)$ & $(8831-210523)$ & $(8240-35070)$ & $(9091-27334)$
\end{tabular}

N-LS, non-lesional skin; ML, melanocytic lesion; CN, compound nevocytic nevi; SN, Spitz nevi; AN, atypical nevi; RGPM, radial growth phase melanoma; VGPM, vertical growth phase melanoma; MMet, melanoma metastases.

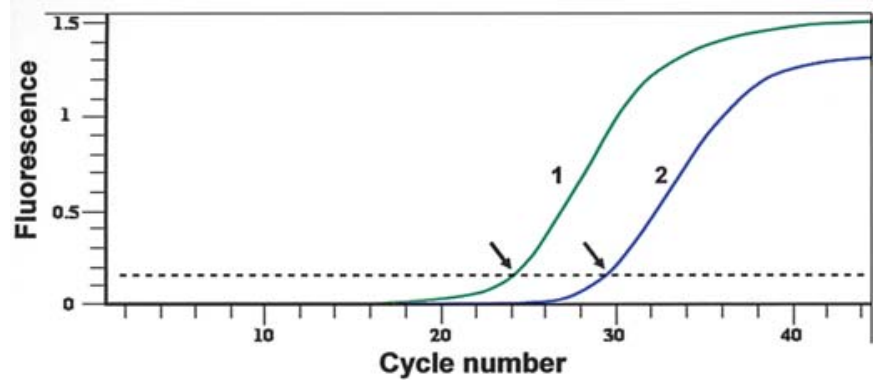

Figure 6. A case of VGPM (1) showing a higher expression level of MIF mRNA than a benign nevocytic nevus (2), as shown by the lower value of PCR cycle number at which the level of fluorescence curve increases $(\mathrm{Ct}$ values, arrows) above the threshold, indicated by the dotted line. as well as 4 out of $12(33.3 \%)$ metastases. Three out of 31 (9.7\%) RGP melanomas, 5 out of $23(21.7 \%)$ VGP melanomas and 2 out of $12(16.6 \%)$ metastases were negative.

MIF $m R N A$. We detected MIF mRNA at different levels of expression in all samples (Fig. 1, Table III). In all groups of melanocytic lesions, the MIF mRNA expression level was significantly higher than in the corresponding tumour-free skin margins $(\mathrm{p}<0.0001)$. The expression level of MIF mRNA tended to be higher in atypical and malignant melanocytic lesions than in benign nevi, although there were some overlapping values. It was significantly lower in all benign nevi $(\mathrm{CN}+\mathrm{SN})$ vs atypical nevi $(\mathrm{p}=0.001)$, vs all melanomas 
$(\mathrm{RGP}+\mathrm{VGP})(\mathrm{p}<0.0001)$, and vs metastases $(\mathrm{p}=0.002)$. The subgroup of compound nevocytic nevi showed a significantly lower expression level of MIF mRNA than atypical nevi $(\mathrm{p}=0.012)$, vertical growth phase melanomas $(\mathrm{p}=0.002)$ (Fig. 6), and metastases ( $\mathrm{p}=0.006)$. MIF mRNA expression was significantly lower in Spitz nevi than in atypical nevi $(\mathrm{p}=0.019)$, and vertical growth phase melanomas $(\mathrm{p}<0.0001)$. There were no significant differences among all the other groups of lesions.

\section{Discussion}

MIF is a unique protein that, acting as a cytokine, hormone, or enzyme, plays a role in inflammatory immune diseases, septic shock and cancer (1). These pleiotropic functions of MIF can partly be attributable to a downstream activation of different, still not completely known, signalling pathways, depending on the cell context and induction state $(2,3,6,15-19)$. Besides the pathways related to its enzymatic activities, both a proliferation-enhancing effect probably mediated through the ERK1/2 pathway, and a JAB1/CSN5/p27Kip1-dependent growth-inhibiting effect have been attributed to MIF $(2,3,6,15-19)$. The recent identification of the cell surface form of the invariant chain (CD74) as a receptor for MIF could increase our knowledge on its mechanism of action in cancer (13). A promoting role of MIF in cancer has been demonstrated in the processes of angiogenesis, cell proliferation, and cell differentiation $(1,3,6-9,15,16)$. This was further supported by experiments showing reduction in tumour size, cell proliferation, secretion of many growth factors and induction of apoptosis after MIF withdrawal/inhibition $(11,31)$. MIF has a role in the regulation of cellular redox stresses and is capable of inhibiting oxidative stress-induced apoptosis (5).

MIF induces immunotolerance, possibly partly due to its known capability of inhibiting NK activity, thus hampering the apoptotic process (11). However, based on the known role of CD74 in antigen presenting, additional MIF receptorrelated pathways can be expected to act in the status of immunotolerance induced by $\operatorname{MIF}(1,3,4,13,15)$. These observations are interesting in cancer such as melanoma, which is characterized by both immunotolerance and an altered redox status, resulting in a low rate of spontaneous and inducible apoptosis that contributes to tumour clinical aggressiveness (40). It is known that, besides inflammatory cells, tumour cells can produce MIF independently (1). Intriguingly, we observed MIF-positive macrophages, usually at the bottom of melanomas, whereas we noticed that, expecially in vertical growth phase melanomas expressing MIF, intratumoural macrophages were often negative to this cytokine. MIF secretion in cancer could result from complex interaction and mutual influences between tumour cells and mononuclear cell infiltrates in situ, which could result in different properties of the cytokine, according to its source and/or cell context. A negative influence on the secretion of MIF by macrophages could be exerted by tumour cells in some advanced melanomas, as it happens in cancer for other cytokines (33). These observations, of course, need further investigation. In cancer, MIF was found to be expressed much more highly in a variety of tumour cells than in corresponding normal cells $(7,16,21-26,28,30)$. MIF protein and its mRNA overexpression have been reported in various cancers, such as lymphoma, myelomonocytic leukemia, prostatic cancer, breast carcinoma, hypophysial and pituitary adenomas, esophageal and head and neck squamous cell carcinoma, colon cancer, lung cancer, and malignant brain tumours (16,20-22,24-30). To our knowledge, MIF has not yet been extensively studied in tissue samples of cutaneous melanocytic tumours, whereas there are some studies on uveal melanoma and cutaneous melanoma cell lines that demonstrate its involvement also in melanoma cell migration, proliferation and angiogenesis in autocrine and paracrine manners $(7,9,23)$. MIF differs from other secreted cytokines in terms of its capability to be constitutively stored within the cytoplasm of many cell types $(1,7,12)$. In agreement with others $(34,35)$, we detected a widespread expression of MIF protein in the epidermis, especially in the basal layers, in the vascular endothelium and in the epithelial and myoepithelial cells of eccrine sweat glands. In addition, we observed a mild to moderate cytoplasmic positivity to MIF in nerves and smooth muscle, a weak positivity in fat cells, and we noticed that MIF stained the nucleus of some cells in the basal layers of the epidermis and adnexa. Melanocytes are known to produce cytokines independently, besides being one of their targets, and cultured normal melanocytes seem to constitutively express MIF protein in their cytoplasm (7). Also, in biopsies of non-lesional skin, the cytoplasm of normal melanocytes was positive to MIF, and we found high levels of MIF protein expression both in benign and in the majority of malignant lesions, whereas MIF mRNA expression levels were usually significantly much higher in the latter. In benign and atypical nevi, as well as in most melanomas and metastases we observed a homogeneous moderate to strong cytoplasmic positivity to MIF. However, in malignant lesions, cytoplasmic MIF expression tended to be more heterogeneous, showing either weaker positive or negative areas, and two lesions were completely negative to MIF. Atypical nevi and most malignant lesions were characterized by a nuclear positivity to MIF, although we detected a nuclear positivity to MIF also in Spitz and blue nevi. We did not notice a correspondence between nuclear and cytoplasmic staining intensity, as observed by others in pituitary adenoma (36).

In some areas of a few malignant lesions, we observed that MIF protein expression was limited to the nuclei, whereas the cytoplasm was negative. It has been suggested that, in order to be biologically functional, a certain amount of newly synthesized MIF protein is required and that it can differ in its characteristics from stored MIF protein (19). This could partly explain the presence of MIF both in normal melanocytes and in tumour cells and the apparent discrepancy that the different MIF mRNA expression in tumours despite their similar protein expression suggests. A relevant difference was observed in MIF protein expression between most benign nevi on one hand and atypical and malignant lesions on the other hand; its subcellular compartmentalisation into the nucleus in the latter groups. In atypical and malignant lesions, there could be a fraction of newly-transcribed RNA, messenger for a newly synthesized MIF protein mainly located in the nucleus where the following unknown mechanisms possibly translocate: specific protein interactions, intracellular shuttling proteins, etc. This, 
however, does not explain the nuclear positivity to MIF in the subgroup of Spitz-blue benign lesions. These observations prompt us to suppose different effects of MIF protein depending both on the cellular context and on its intracellular compartmentalization in melanocytes, the nuclear one being more aptly found in atypical and malignant lesions and/or in distinct subtypes of benign tumours. This suggests that MIF expression could be associated with a different status and/or trigger different downstream effectors and pathways in distinct lesions. These hypotheses need further investigation that should also consider the possible differences that a nucleo-cytoplasmic rather than a cytoplasmic MIF protein expression could bear in terms of prognosis in melanomas. Previous studies demonstrated, in some melanoma cell lines, an increased cytoplasmic MIF expression and the presence of nuclear positivity in comparison to normal cultured melanocytes (7). An increased number of tumour cell nuclei expressing MIF protein in comparison to normal cells has been observed also in other tumours (36), suggesting a role of MIF nuclear location in the process of tumourigenesis and/or as a response to this process. Different pathways resulting in the nuclear compartmentalization of MIF could also be expected to occur in benign versus malignant lesions. Many nuclear and nonnuclear factors can either be modulated by MIF catalytic activities or directly interact and/or be inactivated by MIF protein; among them $\mathrm{p} 53$, JAB1/CSN5, the antioxidant protein PAG, TLR4 as a component of the LPS signaling pathway, AP-1, NF- $\kappa$, and possibly other receptors and kinases $(3,12,17)$. By binding the transcriptional co-activator Jab1 that is overexpressed in the nuclei of some malignant cells, MIF has been shown to moderate Jab1 activity, resulting in a growth-inhibiting effect (16). Interaction with ERK1/2 instead leads to a direct proliferation-enhancing effect $(3,18)$. The possibility that MIF overexpression may contribute to tumour proliferation via p53 inhibition, thus facilitating oncogenic transformation, has been hypothesized in malignant glial tumours, in which a predominantly nuclear expression of MIF was observed (24). Furthermore, it has been found that activated MIF receptor, CD74, acts as a signalling molecule by nuclear activation of $\mathrm{NF}-\kappa \beta(2,12,13)$.

The role and the effects of other known or yet unidentified factors that can be modulated or posttranscriptionally regulated by MIF should be further investigated and elucidated. Therefore, to date, there are conflicting results regarding both the role of MIF in tumourigenesis and its nuclear expression, which have been considered to be associated with the process of oncogenic transformation, predictive either of a better or a poor prognosis, or of no prognostic significance $(22,24,27)$. Intriguingly, we detected MIF-positive nuclei also in benign lesions, particularly in Spitz nevi. Furthermore, nuclear positivity to MIF was also shown by some cells of the adnexa and epidermal basal layer. It is conceivable that MIF nuclear expression in normal epidermis and in some benign melanocytic tumours can be associated with and/or can be a response to the renewal of the basal cell compartment and/or to the proliferative activity in some benign lesions, such as Spitz nevi. In malignant tumours, additional pathways can occur and different factors interact and/or bind to MIF. These hypotheses warrant further investigation that should take into consideration the cell biology, including proliferation/apoptosis, deregulation of other onco-genes, and the role of other epigenetic factors, in addition to the clinical behaviour of the tumour and patient survival.

\section{Acknowledgements}

This study was supported in part by a grant (PAR) from the University of Siena, Italy.

\section{References}

1. Baugh JA and Bucala R: Macrophage migration inhibitory factor. Crit Care Med 30: S27-S35, 2002.

2. Roger T, David J, Glauser MP and Calandra T: MIF regulates innate immune responses through modulation of Toll-like receptor 4. Nature 414: 920-924, 2001.

3. Lue H, Kleemann R, Calandra T, Roger T and Bernhagen J: Macrophage migration inhibitory factor (MIF): mechanisms of action and role in disease: Microb Infect 4: 449-460, 2002.

4. Calandra T and Roger T: Macrophage migration inhibitory factor: a regulator of innate immunity. Nat Rev Immunol 3: 791-800, 2003.

5. Nguyen MT, Lue H, Kleemann R, et al: The cytokine macrophage migration inhibitory factor reduces pro-oxidative stressinduced apoptosis. J Immunol 170: 3337-3347, 2003.

6. Takahashi N, Nishihara J, Sato Y, et al: Involvement of macrophage migration inhibitory factor (MIF) in the mechanism of tumor cell growth. Mol Med 4: 707-714, 1998.

7. Shimizu T, Abe R, Nakamura H, Ohkawara A, Suzuki M and Nishihira J: High expression of macrophage migration inhibitory factor in human melanoma cells and its role in tumor cell growth and angiogenesis. Biochem Biophys Res Commun 264: 751-758, 1999.

8. Nishihira J, Ishibashi T, Fukushima T, Sun B, Sato Y and Todo S: Macrophage migration inhibitory factor (MIF): its potential role in tumor growth and tumor-associated angiogenesis. Ann NY Acad Sci 995: 171-182, 2003.

9. Rumpler G, Becker B, Hafner C, et al: Identification of differentially expressed genes in models of melanoma progression by cDNA array analysis: SPARC, MIF and a novel cathepsin protease characterize aggressive phenotypes. Exp Dermatol 12: 761-771, 2003.

10. Lin JC, Chang SY, Hsieh DS, Lee CF and Yu DS: The association of Id-1, MIF and GSTpi with acquired drug resistance in hormone independent prostate cancer cells. Oncol Rep 13: 983-988, 2005.

11. Apte RS, Sinha D, Mayhew E, Wistow GJ and Niederkorn JY: Cutting edge: role of macrophage migration inhibitory factor in inhibiting NK cell activity and preserving immune privilege. $\mathrm{J}$ Immunol 160: 5693-5696, 1998.

12. Kleemann R, Hausser A, Geiger G, et al: Intracellular action of the cytokine MIF to modulate AP-1 activity and the cell cycle through Jab1. Nature 408: 211-216, 2000.

13. Leng L, Metz CN, Fang Y, et al: MIF signal transduction initiated by binding to CD74. J Exp Med 197: 1467-1476, 2003.

14. Liao H, Bucala R and Mitchell RA: Adhesion-dependent signaling by macrophage migration inhibitory factor (MIF). J Biol Chem 278: 76-81, 2003

15. Mitchell RA: Mechanisms and effectors of MIF-dependent promotion of tumourigenesis. Cell Signal 16: 13-19, 2004.

16. Musat M, Vax VV, Borboli N, Gueorguiev M, Bonner S, Korbonits $\mathrm{M}$ and Grossman $\mathrm{AB}$ : Cell cycle dysregulation in pituitary oncogenesis. Front Horm Res 32: 34-62, 2004.

17. Cao WG, Morin M, Metz C, Maheux R and Akoum A: Stimulation of macrophage migration inhibitory factor expression in endometrial stromal cells by interleukin 1(beta) involving the nuclear transcription factor $\mathrm{NF}(\mathrm{kappa}) \mathrm{B}$. Biol Reprod (In press)

18. Petrenko O and Moll UM: Macrophage migration inhibitory factor MIF interferes with the Rb-E2F pathway. Mol Cell 17: 225-236, 2005.

19. Sun B, Nishihira J, Yoshiki T, Kondo M, Sato Y, Sasaki F and Todo S: Macrophage migration inhibitory factor promotes tumor invasion and metastasis via the Rho-dependent pathway. Clin Cancer Res 11: 1050-1058, 2005. 
20. Nishihira J, Koyama Y and Mizue Y: Identification of macrophage migration inhibitory factor in human leukemia HL-60 cells and its induction by lipopolysaccharide. Biochem Mol Biol Int 40: 861-869, 1996.

21. Bini L, Magi B, Marzocchi B, et al: Protein expression profiles in human breast ductal carcinoma and histologically normal tissue. Electrophoresis 18: 2832-2841, 1997.

22. Kamimura A, Kamachi M, Nishihira J, et al: Intracellular distribution of macrophage migration inhibitory factor predicts the prognosis of patients with adenocarcinoma of the lung. Cancer 89: 334-341, 2000.

23. Repp AC, Mayhew ES, Apte S and Niederkorn JY: Human uveal melanoma cells produce macrophage migration-inhibitory factor to prevent lysis by NK cells. J Immunol 165: 710-715, 2000.

24. Markert JM, Fuller CM, Gillespie GY, et al: Differential gene expression profiling in human brain tumors Physiol Genomics 5: 21-33, 2001.

25. Bando H, Matsumoto G, Bando M, et al: Expression of macrophage migration inhibitory factor in human breast cancer: association with nodal spread. Jpn J Cancer Res 93: 389-396, 2002.

26. Tomiyasu M, Yoshino I, Suemitsu R, Okamoto T and Sugimachi K: Quantification of macrophage migration inhibitory factor mRNA expression in non-small cell lung cancer tissues and its clinical significance. Clin Cancer Res 8: 3755-3760, 2002.

27. Howard BA, Zheng Z, Campa MJ, et al: Translating biomarkers into clinical practice: prognostic implications of cyclophilin A and macrophage migratory inhibitory factor identified from protein expression profiles in non-small cell lung cancer. Lung Cancer 46: 313-323, 2000.

28. Meyer-Siegler Kl, Iczkowski KA and Vera PL: Further evidence for increased macrophage migration inhibitory factor expression in prostate cancer. BMC Cancer 5: 73, 2005.

29. Michael A, Stephan C, Kristiansen G, Burckhardt M, Loening SA, Schnorr D and Jung K: Diagnostic validity of macrophage migration inhibitory factor in serum of patients with prostate cancer: a re-evaluation. Prostate 62: 34-39, 2005.
30. Xia HH, Zhang ST, Lam SK, Lin MC, Kung HF and Wong BC: Expression of macrophage migration inhibitory factor in esophageal squamous cell carcinoma and effects of bile acids and NSAIDs. Carcinogenesis 26: 11-15, 2005.

31. Sasaki Y, Kasuya K, Nishihira J, Magami Y, Tsuchida A, Aoki T and Koyanagi Y: Suppression of tumor growth through introduction of an antisense plasmid of macrophage migration inhibitory factor. Int J Mol Med 10: 579-583, 2002.

32. Watanabe H, Shimizu T, Nishihira J, et al: Ultraviolet Ainduced production of matrix metalloproteinase- 1 is mediated by macrophage migration inhibitory factor (MIF) in human dermal fibroblasts. J Biol Chem 279: 1676-1683, 2004.

33. Chen JJ, Lin YC, Yao PL, et al: Tumor-associated macrophages: the double-edged sword in cancer progression. J Clin Oncol 23: 953-964, 2005.

34. Shimizu T, Ohkawara A, Nishihira J and Sakamoto W: Identification of macrophage migration inhibitory factor (MIF) in human skin and its immunohistochemical localization. FEBS Lett 381: 199-202, 1996.

35. Steinhoff M, Meinhardt A, Steinhoff A, et al: Evidence for a role of macrophage migration inhibitory factor in psoriatic skin disease. Br J Dermatol 141: 1061-1066, 1999.

36. Pyle ME, Korbonits M, Gueorguiev M, et al: Macrophage migration inhibitory factor expression is increased in pituitary adenoma cell nuclei. J Endocrinol 176: 103-110, 2003.

37. Arcuri F, Cintorino M, Vatti R, et al: Expression of macrophage migration inhibitory factor transcript and protein by first trimester human trophoblasts. Biol Reprod 60: 1299-1303, 1999.

38. De Kok JB, Roelofs RW, Giesendorf BA, et al: Normalization of gene expression measurements in tumor tissues: comparison of 13 endogenous control genes. Lab Invest 85: 154-159, 2005.

39. Livak KJ and Schmittgen TD: Analysis of relative gene expression data using Real-Time Quantitative PCR and the $2-{ }^{-\Delta \mathrm{Ct}}$ method. Methods 25: 402-408, 2001.

40. Meyskens FL Jr, McNulty SE, Buckmeier JA, Tohidian NB, Spillane TJ, Kahlon RS and Gonzalez RI: Aberrant redox regulation in human metastatic melanoma cells compared to normal melanocytes. Free Radic Biol Med 31: 799-808, 2001. 